\title{
Things that go bump in the night: From $J / \psi \phi$ to other mass spectrum
}

\author{
Kai $\mathrm{Yi}^{1}$ \\ 1 Department of Physics and Astronomy, University of Iowa, \\ Iowa City, Iowa 52242-1479, USA \\ email: yik@fnal.gov \\ (Dated: June 25, 2018)
}

\begin{abstract}
This article summarizes a brief development of exotic mesons with Vector-Vector (VV) final states starting from the $J / \psi \phi$ mass spectrum, as well as extensions to other final states for stronginteraction physics and physics beyond standard model. These VV final states are very suitable to be studied at CEPC through associate production and two-photon production, as well as a visionary low energy photon collider.

PACS numbers:
\end{abstract}

\section{INTRODUCTION}

The existence of potential multiple structures in the $J / \psi \phi$ mass spectrum [1] was in question several years ago, reference [2] summarized the status of $J / \psi \phi$ mass spectrum and the Vector-Vector (VV) final states by then, and proposed to search for structures near threshold in the VV systems composed entirely of c quarks and $\mathrm{b}$ quarks. Now the first two structures in the $J / \psi \phi$ mass spectrum, renamed as $X(4140)$ and $X(4274)$ by PDG, have been confirmed or re-confirmed by CMS [3], D0 [4] and LHCb [5], in addition the LHCb collaboration announced two more new structures, named as $X(4500)$ and $X(4700)$ in the same mass spectrum. Both BaBar and BES collaborations searched the $J / \psi \phi$ mass spectrum without significant signals [6]. These states are the first of such kind of exotic meson candidates that have no light quark components, and added an important piece to the exotic meson family. There are total five structures reported in the $J / \psi \phi$ mass spectrum adding the $X(4350)$ spotted in the two-photon process at Belle experiment [7. The $J^{P C}$ possibilities of $1^{-+}, 1^{++}$and $3^{-+}$are excluded for $X(4350)$ inferred from its production process. Assuming $X(4350)$ is real, it is suppressed in the B decays in the case of a spin 2 state. There is also large difference for the width of the $X(4140)$ measued by LHCb experiment and other experiments [13. More statistics from LHC and Belle II will help to make things clear. These states demand a through theoretical understanding eventually. Possible interpretations have been developed over years, however, we do not have a perfect model or explanation for these $J / \psi \phi$ structures.

A natural extension for the $J / \psi \phi$ mass spectrum would be the $\psi(2 S) \phi$ system. The CMS collaboration has observed the relevant decays $-B^{ \pm} \rightarrow \psi(2 S) \phi K^{ \pm}$, however, due to limited statistics and the limited available phase space allowed for $\psi(2 S) \phi$ mass in the B decays, CMS did not exam the $\psi(2 S) \phi$ mass spectrum in this decay [8]. Other VV systems, include $\psi \psi, \psi \Upsilon, \Upsilon \Upsilon$, can be further explored at LHC with large data sample, where $\psi$ and $\Upsilon$ can be either on-shell or off-shell. The leptonic decays of these vector states provide many advantages for investigation, especially at ATLAS and CMS experiments that have no good hadron particle identification power-but both experiments have excellent lepton identification performance and momentum resolution, as well as excellent mass resolution. The production cross section of $J / \psi J / \psi$ has been measured at LHC experiments at various energies 91, and recently CMS measured the production cross section of $\Upsilon \Upsilon$ at $\sqrt{S}=8 \mathrm{TeV}$ for the first time [10. The production of $J / \psi \Upsilon$ at $\sqrt{S}=8 \mathrm{TeV}$ has been preliminarily investigated in a thesis work [11. These benchmark measurements show evidence that there are enough statistics to search for possible structures in these VV systems composed entirely of c quark and b quarks.

A recent preliminary investigation through a thesis work [12] shows an evidence of a structure at $18 \mathrm{GeV}$ in the $\Upsilon(1 S) \ell^{+} \ell^{-}$final states with a width less than $150 \mathrm{MeV}$. The observed mass is several hundreds $\mathrm{MeV}$ below the threshold of double $\eta_{b}$ mass, which is the first one among the observed $\mathrm{X} / \mathrm{Y} / \mathrm{Z}$ states, the others are either above or near the threshold of two particles. Assuming it as a real physics effect, one possibility is that this is a new state decaying to one on-shell and one off-shell $\Upsilon(1 S)$, which is another VV system with V being on-shell or off-shell. This article focuses on possibilities of investigating the VV system at various existing or visionary colliders in the world. 


\section{MOTIVATION}

A recent summary of the discovered $\mathrm{X} / \mathrm{Y} / \mathrm{Z}$ states in the last decade can be found at Ref. [13], and the recent theoretical developments of understanding of these $\mathrm{X} / \mathrm{Y} / \mathrm{Z}$ states can be found at Ref. 14. The nature of these states is far away from a complete understanding, despite of the fact that many models such as hadrocharmonium, molecule, CUSP etc have been proposed to explain. As mentioned in the introduction section, there is one kind of candidates that decays to VV mesons without light quark component among the newly discovered exotic candidate zoo, where $\mathrm{V}$ can be either an on-shell or off-shell vector meson. These VV candidates are not understood and there is little information known from both production and decay aspects. On the other hand, these VV system can be produced through two-photon collision, and this article provides preliminary investigation of decaying kinematic distributions at CEPC [18 and a visionary photon collider. In addition, possible associate production at CEPC is also discussed.

As for the $18 \mathrm{GeV}$ excess reported in the thesis work [12], several possibilities exist. Assuming this is due to real physics effects, a simple naive explanation would be a bound state composed of tetra-bottom quark which is analog to positronium molecule state [15]. Theoretical development along this direction can be found at Ref [16. However, a recent lattice QCD calculation denies the existence of a tetra-bottom quark state [17. Other possibilities include a light scalar or pseudo-scalar state. Nevertheless, the region below the Z mass has never been fully explored, it is worth to investigate as a general case with some efforts. This article discusses possible exploration in this mass region at various existing or visionary colliders, the focus is on the kinematic distributions as a general phase space decay.

\section{Possible decay channels to explore}

As stated above, it is not thoroughly investigated for the region below the $\mathrm{Z}$ mass, there is potential for new composite or elementary particles existing in this region, thus we assume there are possible new states in this region, and study the kinematics in various decay channels, and in various facilities. Assuming a new state existing below the $\mathrm{Z}$ mass, the possible decay channels are different and the relative ratios among various channels are also different, depending on what it is. For instance, a typical hadronic state such as a tetra-bottom (tetra-charm) quark state would prefer to decay into bottomonium (charmonium) related final states, on the other hand, a typical new state that is an extension of standard model may choose to decay to $\gamma \gamma$ or $j j$. There are possibilities that both hadronic states and elementary states can exist in this mass region, thus we consider the following decay channels as a general case: $V V$ where $\mathrm{V}$ can be an on-shell or off-shell vector meson; $V \gamma ; \ell^{+} \ell^{-} ; \gamma \gamma ; j j$ etc. The possible new state masses assumed in this article are $18.5,30$ and $50 \mathrm{GeV}$.

- $\mathrm{V}+\mathrm{V}$, where $\mathrm{V}$ is an on-shell or off-shell vector meson with $J^{P C}=1^{--}$such as $J / \psi, \psi(2 S), \Upsilon(n S)$ etc. These channels can be done at the current ATLAS/CMS experiments with V decays to dilepton final states. Depends on the lepton kinematics at different mass regions, this can be difficult for low momentum leptons due to pileup effects. For instance, the peak luminosity at CMS varies from $1 \mathrm{~Hz} / \mathrm{nb}$ in 2011 to $22 \mathrm{~Hz} / \mathrm{nb}$ in 2017 [19], and it is going to be much higher in the future which makes the pileup effect more serious. It is possible to improve the performance with improved detector technology such as timing separation for the charged particles. On the other hand, these channels can be fully investigated at LHCb when enough data samples are accumulated by taking advantage of its relative low peak luminosity (lower pileup).

- $\mathrm{V}+\gamma$, where $\gamma$ can be a reconstructed photon object, or a converted photon reconstructed using trackers, or a virtual gamma decay. Considering the background rate, fake rate and low efficiency for low momentum photons, converted photons seem more plausible for ATLAS/CMS by taking advantage of the vertex information at the conversion point.

- $\gamma \gamma, q \bar{q}, \ell^{+} \ell^{-}$where $\ell=\tau, \mu, e$. These channels are generally difficult to do at the LHC facilities due to trigger restriction, reconstructing challenge and high background rate. However, they can be performed relatively easy at other facilities such as CEPC. Several examples will be demonstrated in the following sections. 


\section{INVESTIGATION AT CEPC FACILITY}

The Circular Electron Positron Collider (CEPC) is a collider being proposed to study the Higgs boson in precision at the collision energy of $250 \mathrm{GeV}$ [18. However, this facility can be used to study many other physics, and this article discusses the possible investigation of the VV system at this $e^{+} e^{-}$collider. The physics tool used here is Pythia8 [20], and the detector simulation tool used is Delphes [21, the specific configuration of the detector used is the embedded CEPC detector in Delphes tool. All decays are simply phase space kinematics. The VV system can not be produced through $e^{+} e^{-}$annihilation because $e^{+} e^{-}$typically annihilate to a virtual photon which is a $1^{--}$object. The VV system, which may come from a new state decays, can be produced at CEPC through two processes: 1) associate production-it can be produced associated with an object such as a $\mathrm{Z}$ or a photon. 2) two-photon process-both electron and positron radiate a virtual photon and the two photons collide to produce it. In principle, this can also be searched and studied inclusively, but we skip the discussion.

There is no detailed cross section information available yet for various production mechanisms, though it can be inferred from Ref. 22] for two-photon production, the author is looking for possible collaboration with theorists to investigate production rate in a through way with different assumptions.

\section{Associate production}

There are two ways to search for a new object with associate production at CEPC. One is to look for the recoiled mass against a fully reconstructed object such as a $\mathrm{Z}$ boson, another way is to investigate a specifically reconstructed final state, which can include a fully reconstructed associate object or can be inclusive. This section discusses the two methods to investigate new objects.

With Recoil technique

The benefit to investigate the recoiled mass is that it includes the object's all final states, which provides large rate to be more plausible to observe, but it is affected by mass resolution and background. The branching fraction for a specific channel can also be measured if a specific channel is reconstructed in the recoil side. Figure 1 shows the fully reconstructed $\mathrm{Z}\left(e^{+} e-\right)$ mass and the recoiled mass against the $\mathrm{Z}$ boson at different collision energies with different object masses: $18.5,30$ and $50 \mathrm{GeV}$. The $\mathrm{Z}$ mass resolution is more or less the same at different collision energies as shown in Fig. 1 (top and bottom left). Figure 1 (top right) shows the recoiled mass distributions with different mass values at collision energy of $250 \mathrm{GeV}$. The mass resolution varies from several $\mathrm{GeV}$ at low mass region to less than $1 \mathrm{GeV}$ at higher mass region. Note that the mass resolution becomes better with higher mass objects due to the squeezed phase space. The mass resolution can be greatly improved in the low mass region with lower collision energy as shown in Fig. 1 (bottom right), this can be done with the designed low collision energy at the beginning.

As we will discuss in the next section, the new object can also be fully reconstructed with a specific channel. Combining with the information with recoiled mass measurement, the branching fraction for a specific channel can be measured.

\section{With fully reconstructed objects}

Here we choose the following specific channels as examples to demonstrate their kinematic distributions at CEPC with phase space decays.

- $e^{+} e^{-} \rightarrow Z X ; X \rightarrow Y(1 S) \mu^{+} \mu^{-} ; Y(1 S) \rightarrow \mu^{+} \mu^{-}$. Figure 2 shows the reconstructed mass, $\eta, p_{T}, p_{Z}$ distributions for a potential $18 \mathrm{GeV}$ object and its decay particles for this process. Due to the recoiling boost from $\mathrm{Z}$ boson in the transverse plane, this object has large transverse momentum up to $110 \mathrm{GeV}$, this feature can be used to select events to reduce background in the final state. The muons in the final states are mostly distributed in the detector's central region. Figure 3 and 4 show the six dimuon mass distributions and the six $\Delta R$ distributions between the six dimuon pairs. The four muons are close to each other against the $\mathrm{Z}$ boson. As one can see, there are mispaired real upsilon in the other dimuon pairs: $(1,4)$ and $(2,3)$, where $\mu_{1}$ and $\mu_{2}$ form the on-shell $\Upsilon(1 S)$ particle and $\mu_{3}$ and $\mu_{4}$ form the second dimuon pair. 
- $e^{+} e^{-} \rightarrow Z X ; X \rightarrow \gamma \gamma$. Figure 5 shows the reconstructed mass, $p_{T}, \eta, \Delta \eta$ and $\Delta \phi$ distributions for a potential $18 \mathrm{GeV}$ object and its decaying particles. Due to the boost from $\mathrm{Z}$ boson in the transverse plane, this object has large transverse momentum up to $110 \mathrm{GeV}$, and the high $p_{T}$ photon has relative flat transverse momentum up to $100 \mathrm{GeV}$, which can be used to optimize the signal in the final state. The photons in the final states are mostly distributed in the detector's central region with close distance in $R$ and $\phi$ spaces.

- $e^{+} e^{-} \rightarrow Z X ; X \rightarrow \tau^{ \pm} \tau^{\mp} ; \tau^{ \pm} \rightarrow \ell^{ \pm}+$anything-else, $\tau^{\mp} \rightarrow \ell^{\mp}+$ anything-else, where $\ell=\mu, e$. Figure 6 shows the reconstructed $\mu^{ \pm} e^{\mp}, \mu^{ \pm} \mu^{\mp}$ and $e^{ \pm} e^{\mp}$ mass distributions, $p_{T}, \eta, \Delta \eta$ and $\Delta \phi$ distributions for the dilepton system. Due to the missing neutrino particles, the reconstructed dilepton masses are not peaked at $18.5 \mathrm{GeV}$ but with a wide mass spectrum below $18.5 \mathrm{GeV}$. The transverse momentum is soften by missing neutrino particles in the final state. The dilepton system and each lepton are mostly distributed in the detector's central region with close distance in $R$ and $\phi$ spaces. This channel may be only possible in $e^{+} e^{-}$machines due to its low background.
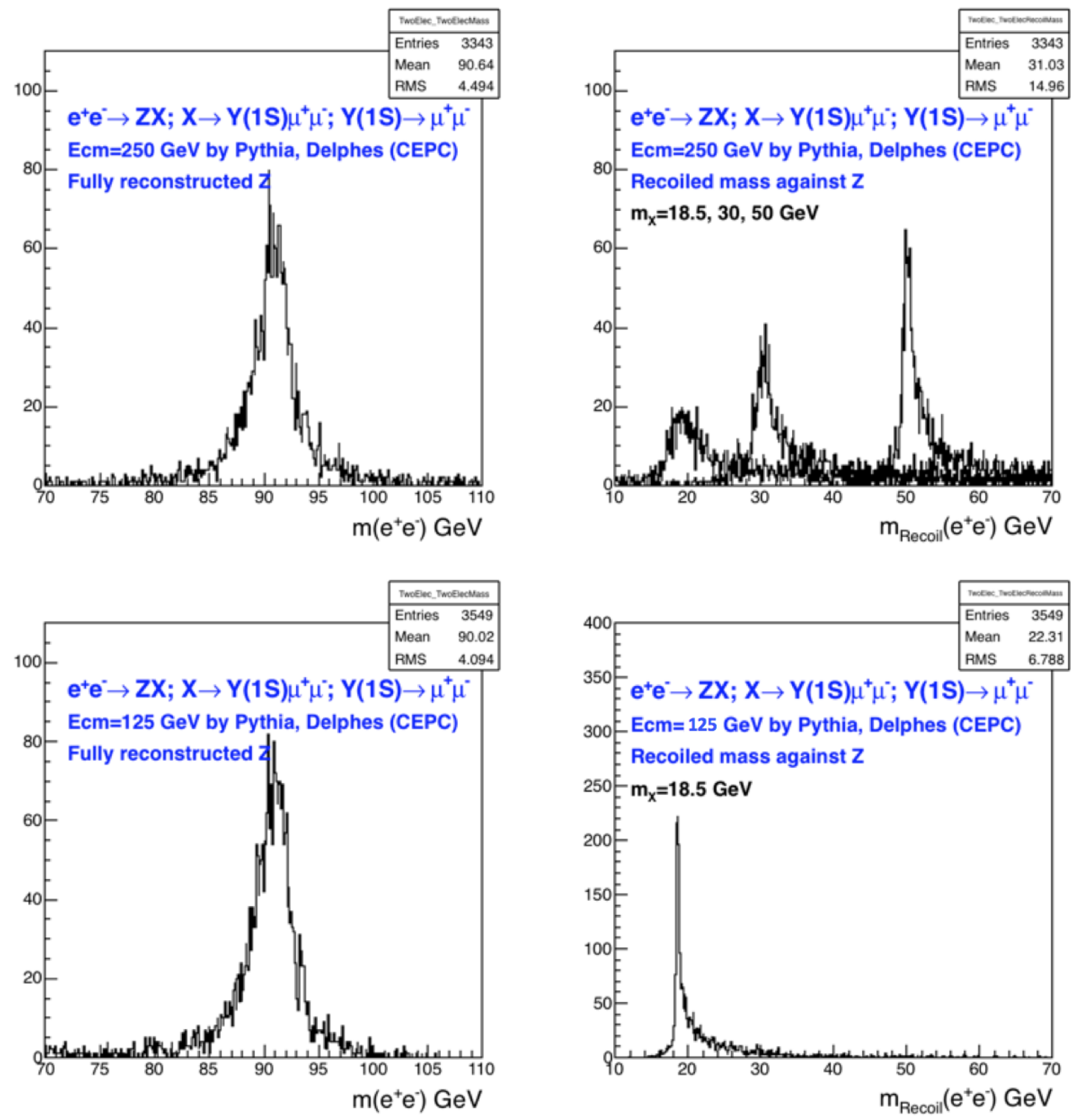

FIG. 1: The fully reconstructed $\mathrm{Z}$ mass and its recoiled mass against $\mathrm{Z}$ particle at CEPC with different collision energy by assuming $X$ particle mass as $18.5,30$ and $50 \mathrm{GeV}$ produced in the process of $e^{+} e^{-} \rightarrow Z X ; X \rightarrow Y(1 S) \mu^{+} \mu^{-} ; Y(1 S) \rightarrow \mu^{+} \mu^{-}$. These events are simulated by Delphes with CEPC configuration. 

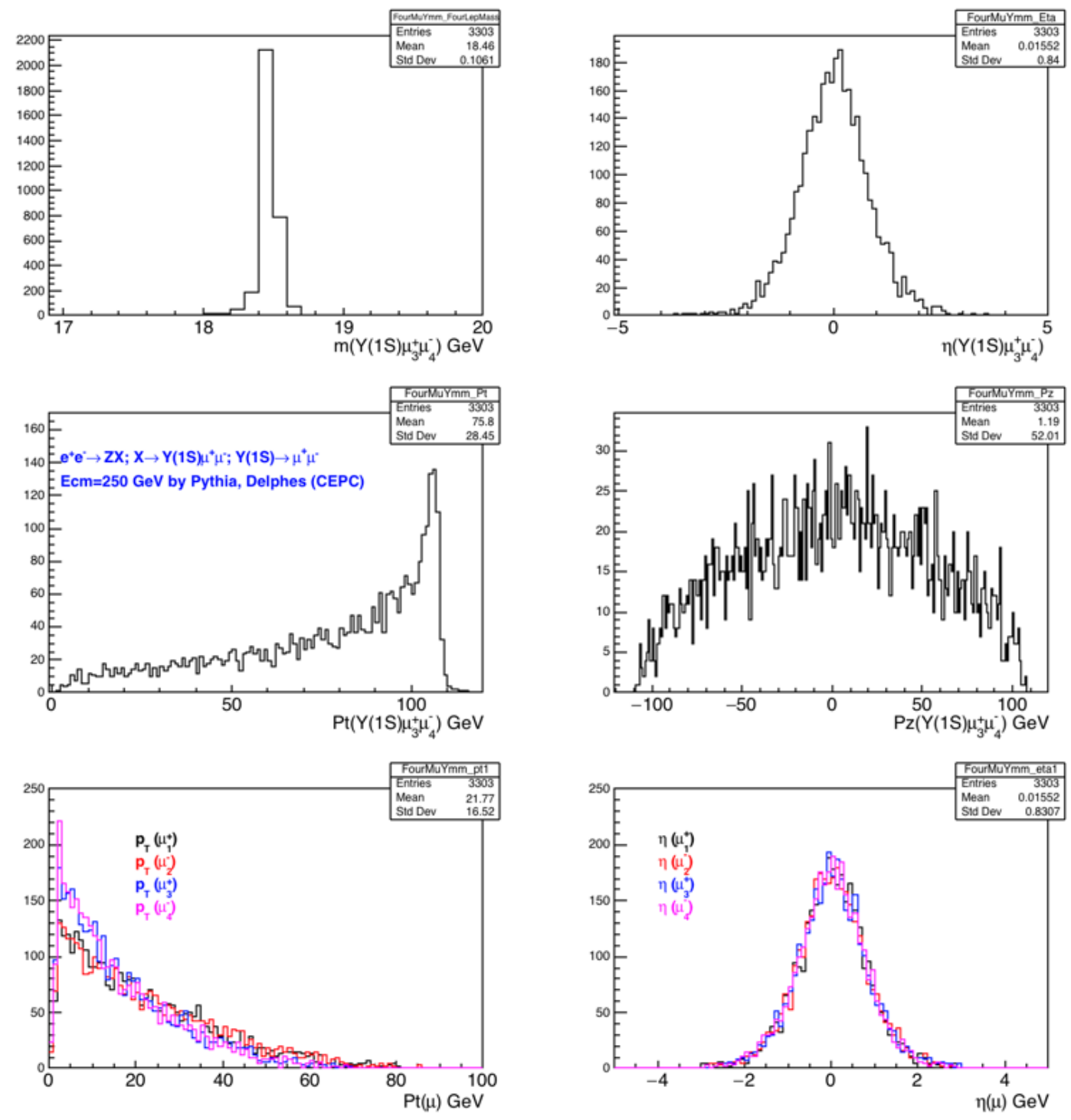

FIG. 2: The features of the four-muon system produced in $e^{+} e^{-} \rightarrow Z X ; X \rightarrow Y(1 S) \mu^{+} \mu^{-} ; Y(1 S) \rightarrow \mu^{+} \mu^{-}$process at CEPC: (top left) The invariant mass of the four muons; (top right) The $\eta$ distribution of the four muons; (middle left) $p_{T}$ of the four muons; (middle right) $p_{Z}$ of the four muons; (bottom left) $p_{T}$ of each muon. (bottom right) $\eta$ of each muon. These events are simulated by Delphes with CEPC configuration. 

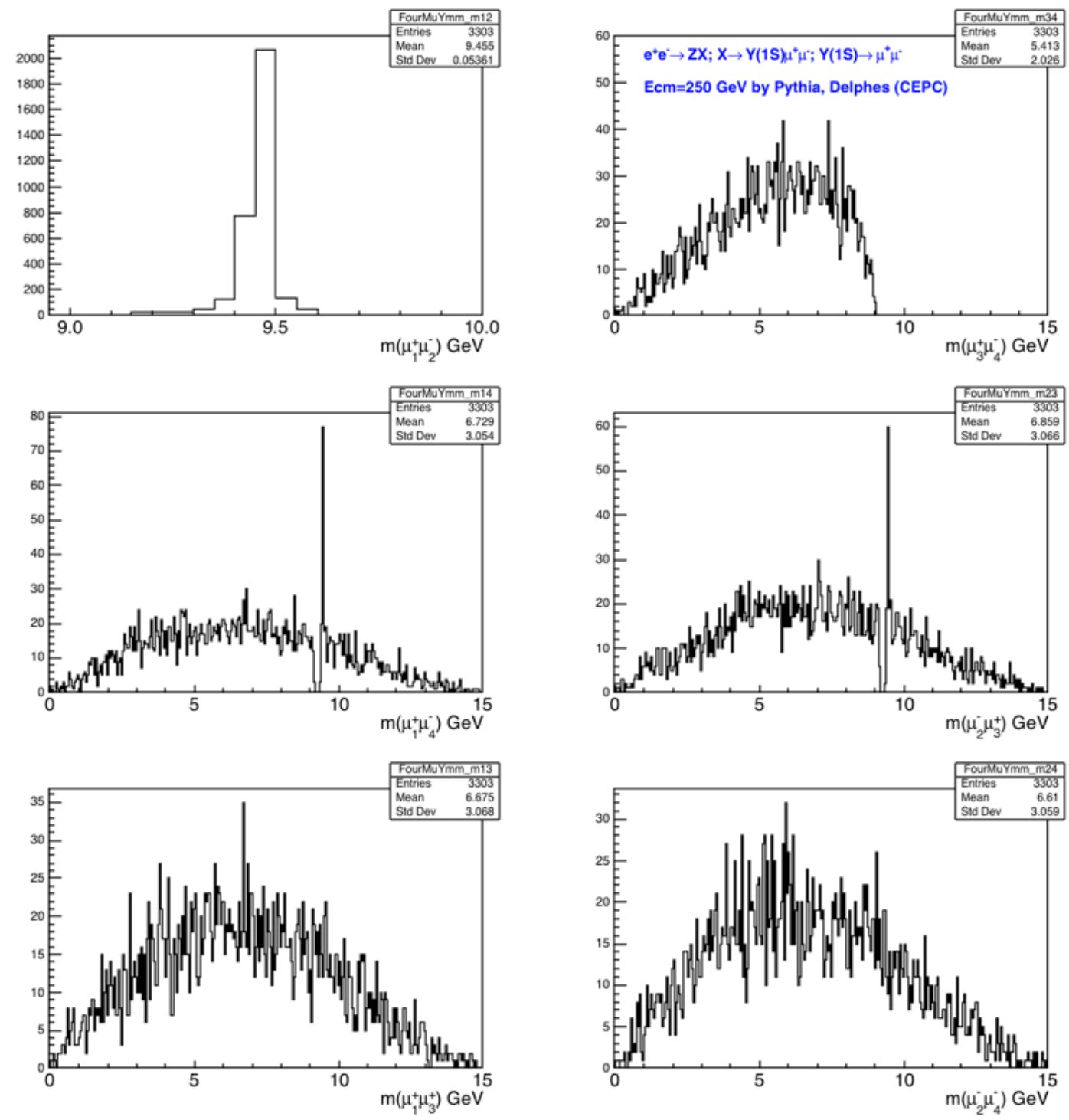

FIG. 3: The six dimuon mass distributions of the four-muon system in the $e^{+} e^{-} \rightarrow Z X ; X \rightarrow Y(1 S) \mu^{+} \mu^{-} ; Y(1 S) \rightarrow \mu^{+} \mu^{-}$ process at CEPC. 

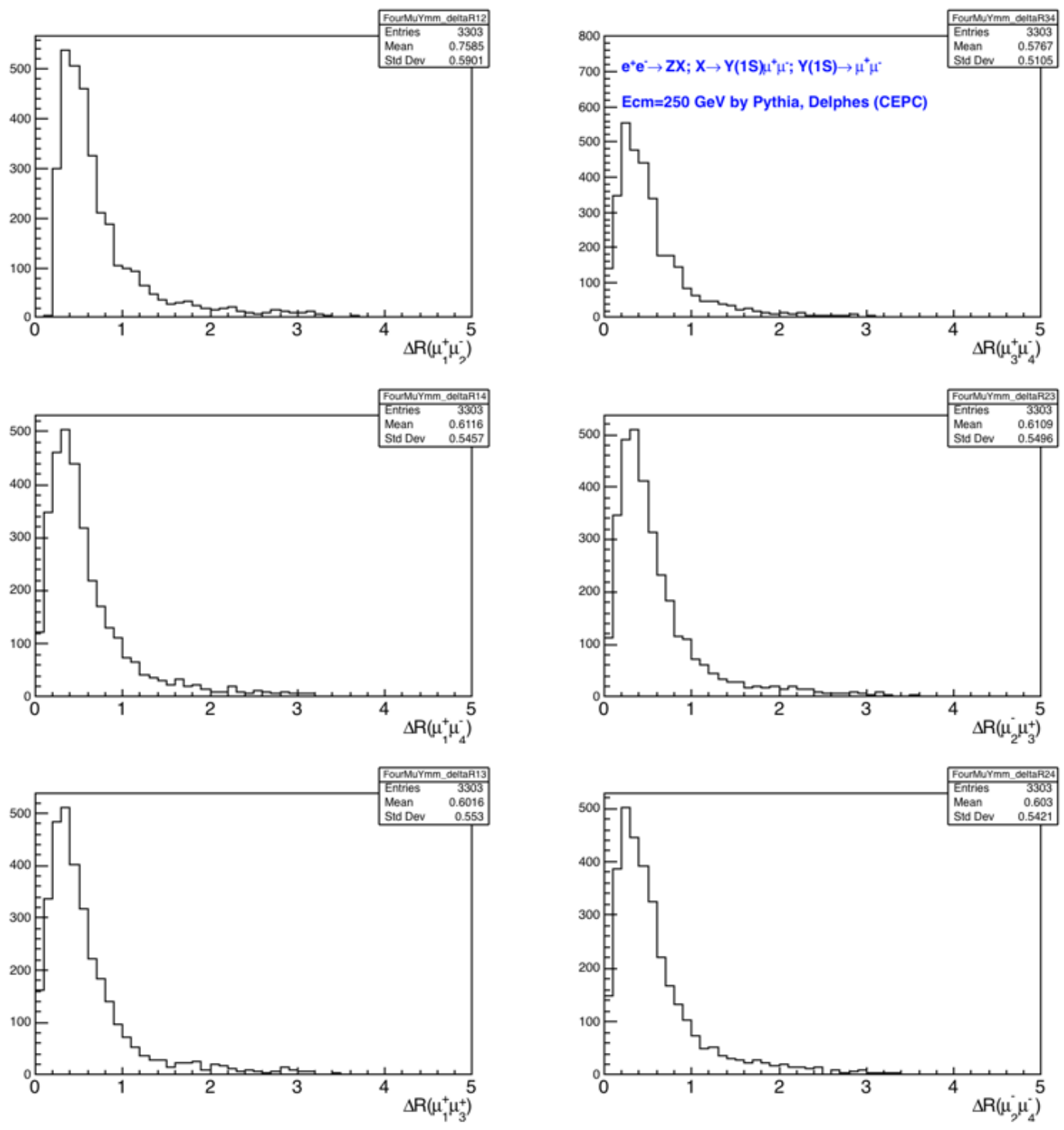

FIG. 4: The six $\Delta R$ distributions of the four-muon system in the $e^{+} e^{-} \rightarrow Z X ; X \rightarrow Y(1 S) \mu^{+} \mu^{-} ; Y(1 S) \rightarrow \mu^{+} \mu^{-}$process at CEPC. 

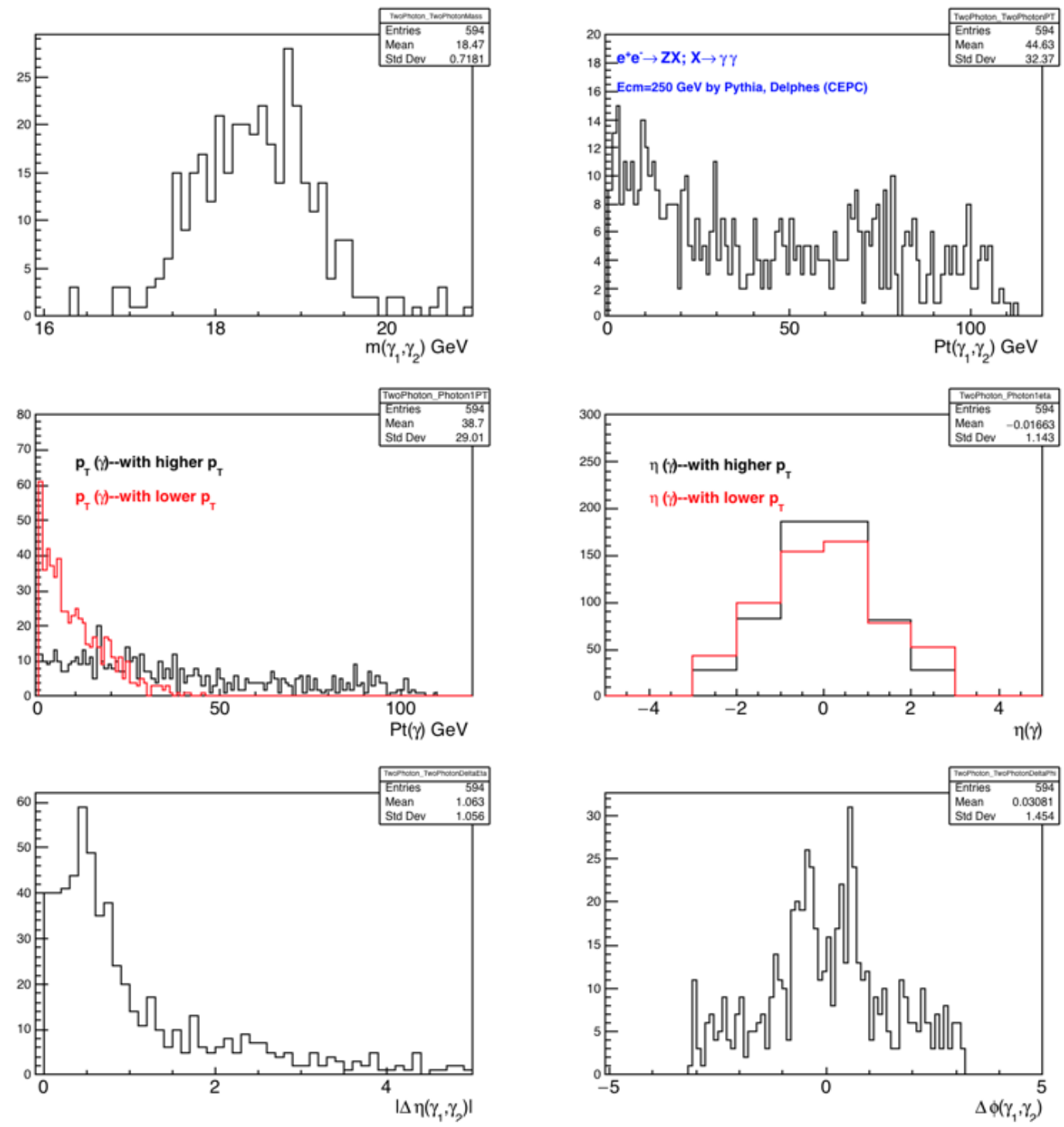

FIG. 5: The features of the $\gamma \gamma$ system in the $e^{+} e^{-} \rightarrow Z X ; X \rightarrow \gamma \gamma$ process at CEPC: (top left) The di-photon mass distribution; (top right) The $p_{T}$ distributions of the di-photon; (middle left) The $p_{T}$ distribution of each photon; (middle right) The $\eta$ distribution of each photon; (bottom left) The $\Delta \eta$ distribution of the di-photon; (bottom right) The $\Delta \phi$ distribution of the di-photon. These events are simulated by Delphes with CEPC configuration. 

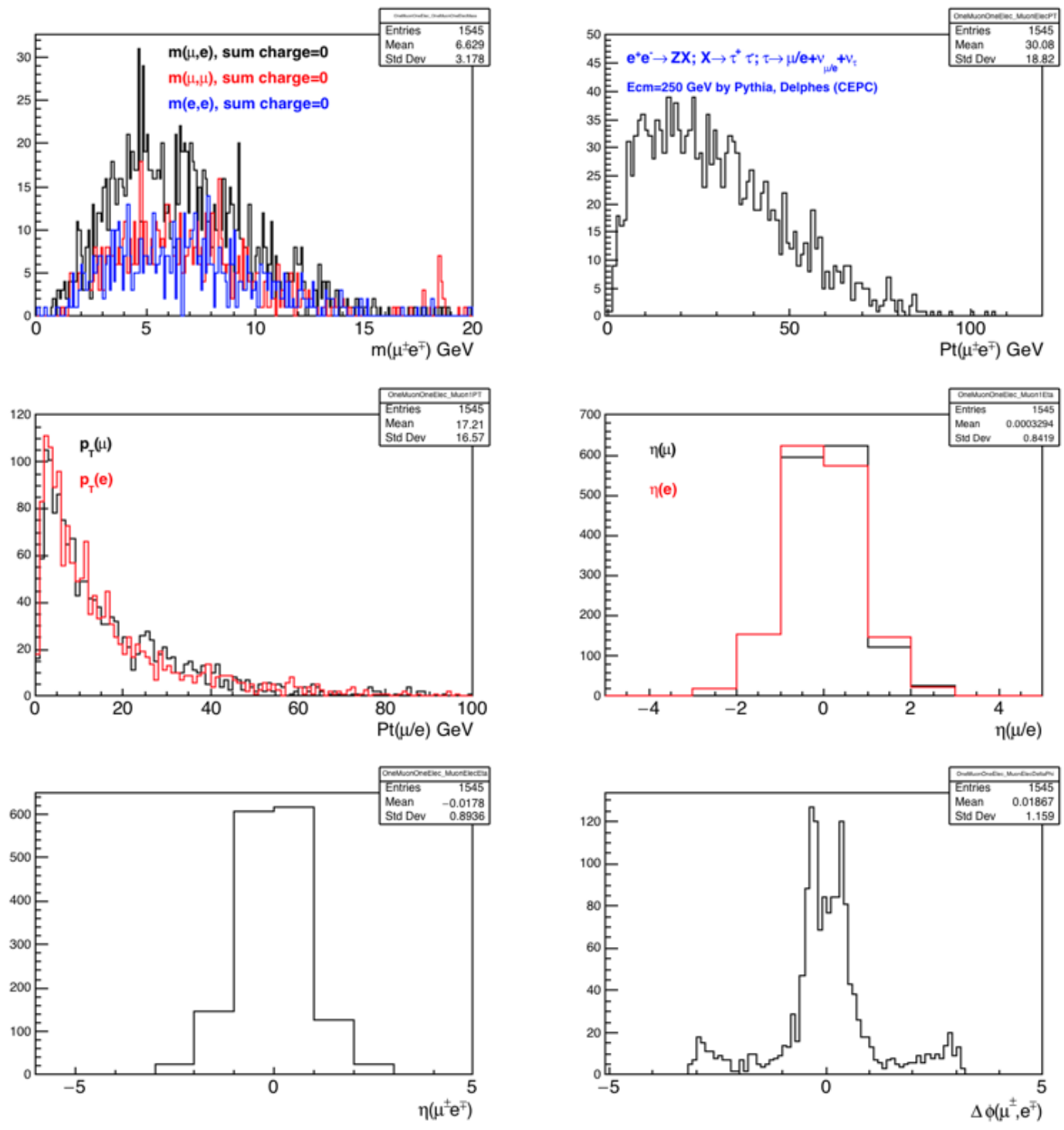

FIG. 6: The features of the $\mu^{ \pm} e^{\mp}\left(\tau^{ \pm} \tau^{\mp}\right)$ system in the $e^{+} e^{-} \rightarrow Z X ; X \rightarrow \tau^{ \pm} \tau^{\mp} ; \tau^{ \pm} \rightarrow \ell^{ \pm}+$anything - else, $\tau^{\mp} \rightarrow$ $\ell^{\mp}+$ anything - else, where $\ell=\mu$,e process at CEPC: (top left) The $\mu^{ \pm} e^{\mp} / \mu^{ \pm} \mu^{\mp} / e^{ \pm} e^{\mp}$ mass distributions; (top right) The $p_{T}$ distributions of $\mu^{ \pm} e^{\mp}$; (middle left) The $p_{T}$ distribution of muon and electron; (middle right) The $\eta$ distribution of muon and electron; (bottom left) The $\eta$ distribution of $\mu^{ \pm} e^{\mp}$; (bottom right) The $\Delta \phi$ distribution between muon and electron. These events are simulated by Delphes with CEPC configuration. 


\section{Two-photon production}

Another possible production for potential new states at CEPC is via the two-photon process: $e^{+} e^{-} \rightarrow$ $e^{+} e^{-} \gamma^{*} \gamma^{*}, \gamma^{*} \gamma^{*} \rightarrow X$. A striking feature of the two-photon process is that the reconstructed object shows "zero" transverse momentum and it is typically used as a selection criteria to separate signal events from background events. However, particles with $J^{P C}$ of $1^{-+}, 1^{++}$and $3^{-+}$are restricted in two-photon process. Three specific channels at CEPC produced through two-photon process are discussed below.

- $e^{+} e^{-} \rightarrow e^{+} e^{-} X ; X \rightarrow Y(1 S) \mu^{+} \mu^{-} ; Y(1 S) \rightarrow \mu^{+} \mu^{-}$. Figure 7 shows the reconstructed mass, $\eta, p_{T}, p_{z}$ and $\phi$ distributions for a potential $18 \mathrm{GeV}$ object and its decay particles from the two-photon production. Since it is singly produced and there is no recoiling boost from associated particles in the transverse plane, the object has zero transverse momentum, and the transverse momentum of its decay particles are low. The object is distributed in the forward region although its decay particles are in the central region along one side. Figure 8 shows the six $\Delta R$ distributions between the six pairs, they are well separated between each other, unlike the distributions from the associate production.

- $e^{+} e^{-} \rightarrow e^{+} e^{-} X ; X \rightarrow \gamma \gamma$. Figure 9 shows the reconstructed mass, $p_{T}, \eta$, and $\phi$ distributions for a potential 18 $\mathrm{GeV}$ object and its decaying particles. As a general feature from two-photon process, this object has a close to zero transverse momentum distribution, thus the photon transverse momentum distributions peak at high end around $9 \mathrm{GeV}$ to make the object's mass. The two decaying photons are back-to-back in $\phi$ plane, and close in $\eta$ plane along one side, thus distributed in the forward region.

- $e^{+} e^{-} \rightarrow e^{+} e^{-} X ; X \rightarrow \tau^{ \pm} \tau^{\mp} ; \tau^{ \pm} \rightarrow \ell^{ \pm}+$anything - else, $\tau^{\mp} \rightarrow \ell^{\mp}+$ anything - else, where $\ell=\mu, e$. Figure 10 shows the reconstructed $\mu^{ \pm} e^{\mp}, \mu^{ \pm} \mu^{\mp}$ and $e^{ \pm} e^{\mp}$ masses, $p_{T}, \eta, \Delta \eta$ and $\Delta \phi$ distributions for the dilepton system. The dilepton mass distributions are the same as that in the associate production: widely peak below $18.5 \mathrm{GeV}$. The dilepton system is distributed in the forward region but each lepton is mostly distributed in the detector's central region along one side and back-to-back in $\phi$ spaces. Although it is diluted for the dilepton transverse momentum due to missing neutrino particles in the final state, the dilepton transverse momentum distribution is still concentrated at low momentum end (around $2 \mathrm{GeV}$ ), this can be used to separated signal events from background events. 

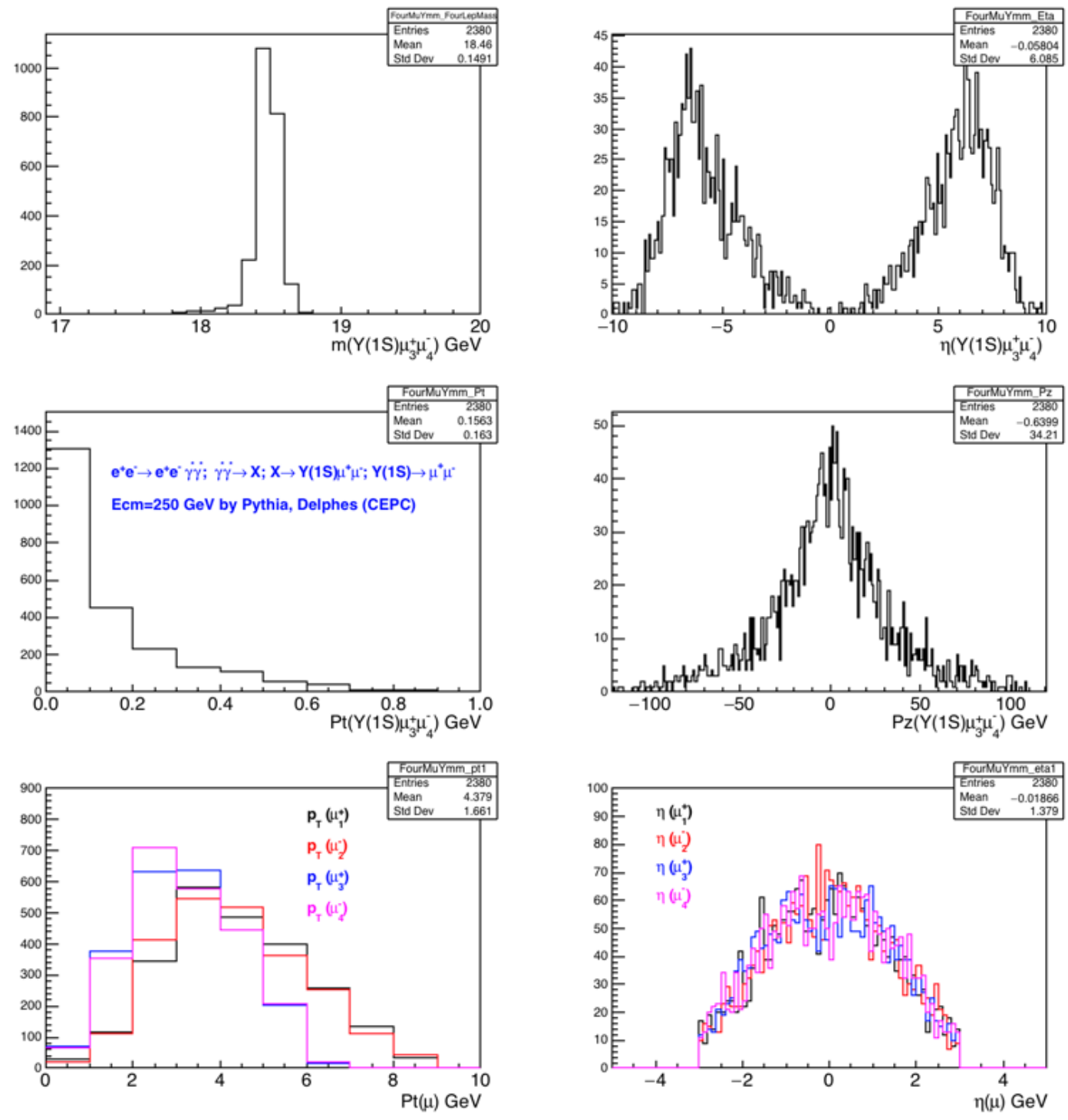

FIG. 7: The features of the four-muon system in the $e^{+} e^{-} \rightarrow e^{+} e^{-} X ; X \rightarrow Y(1 S) \mu^{+} \mu^{-} ; Y(1 S) \rightarrow \mu^{+} \mu^{-}$process at CEPC: (top left) The invariant mass of the four muons; (top right) The $\eta$ distribution of the four muons; (middle left) $p_{T}$ of the four muons; (middle right) $p_{Z}$ of the four muons; (bottom left) $p_{T}$ of each muon. (bottom right) $\eta$ of each muon. These events are simulated by Delphes with CEPC configuration. 

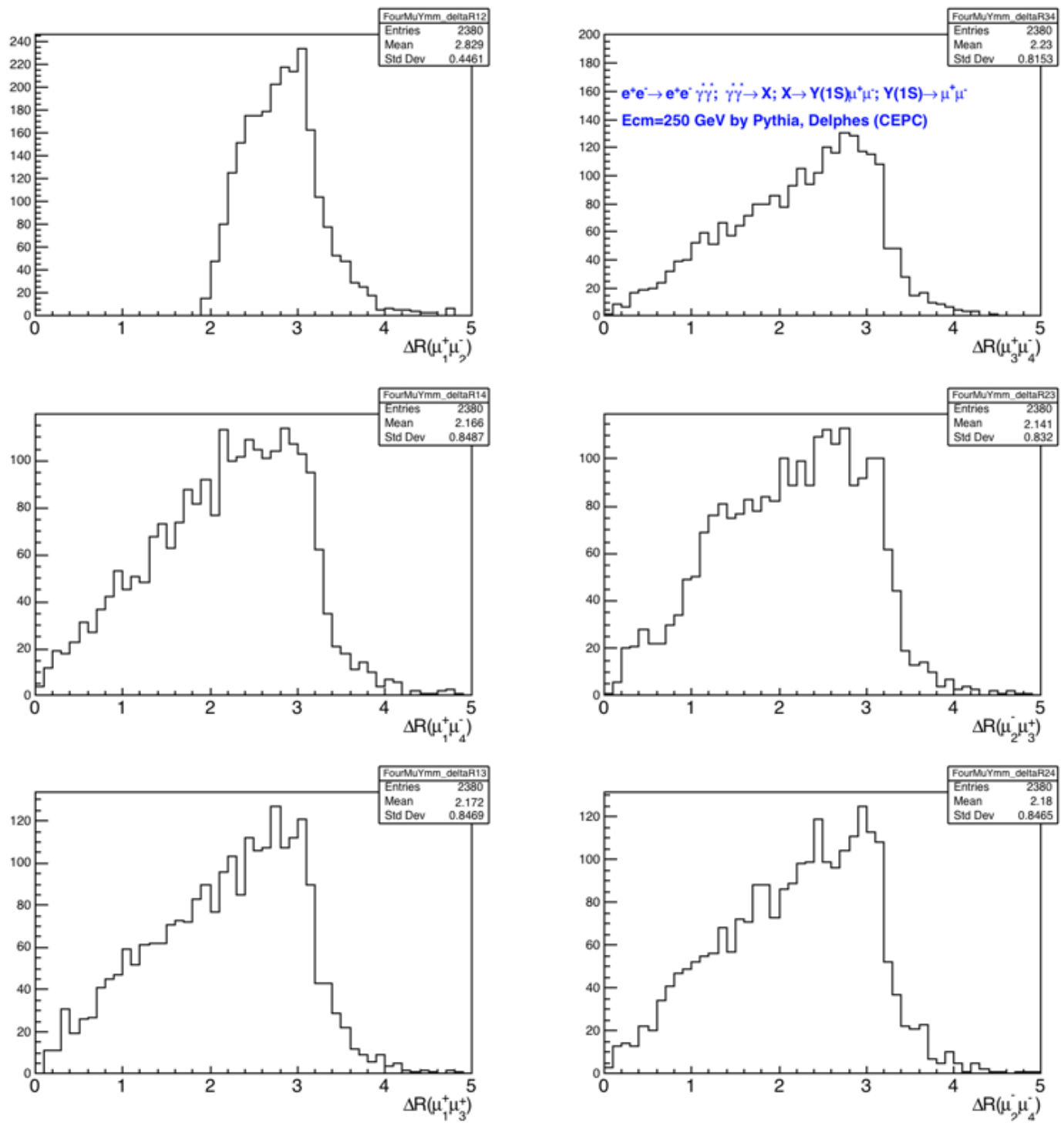

FIG. 8: The six $\Delta R$ distributions of the four-muon system in the $e^{+} e^{-} \rightarrow e^{+} e^{-} X ; X \rightarrow Y(1 S) \mu^{+} \mu^{-} ; Y(1 S) \rightarrow \mu^{+} \mu^{-}$process at CEPC. 

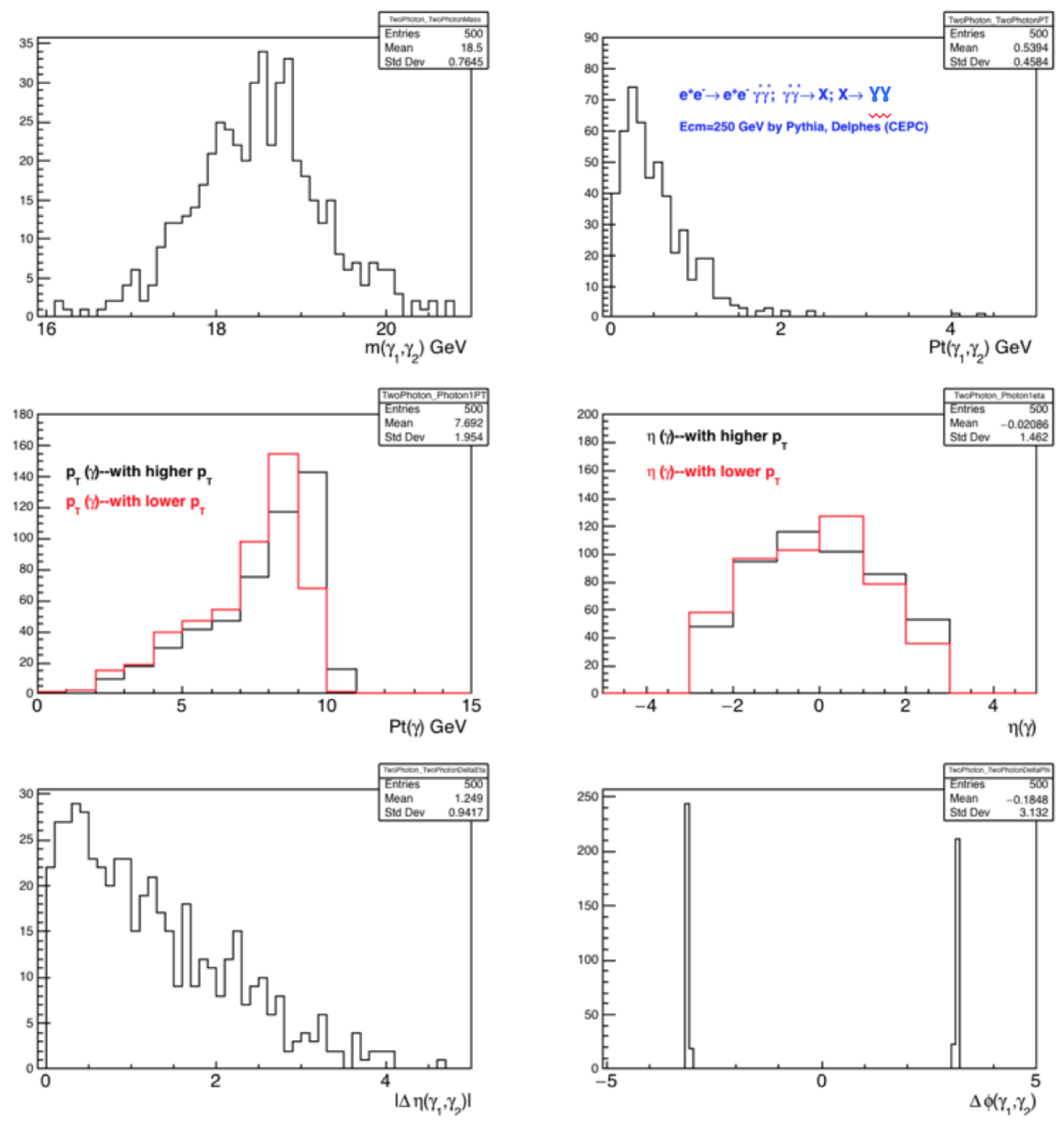

FIG. 9: The features of the $\gamma \gamma$ system in the $e^{+} e^{-} \rightarrow e^{+} e^{-} X ; X \rightarrow \gamma \gamma$ process at CEPC: (top left) The di-photon mass distribution; (top right) The $p_{T}$ distribution of the di-photon; (middle left) The $p_{T}$ distribution of each photon; (middle right) The $\eta$ distribution of each photon; (bottom left) The $\Delta \eta$ distribution of the di-photon; (bottom right) The $\Delta \phi$ distribution of the di-photon. These events are simulated by Delphes with CEPC configuration. 

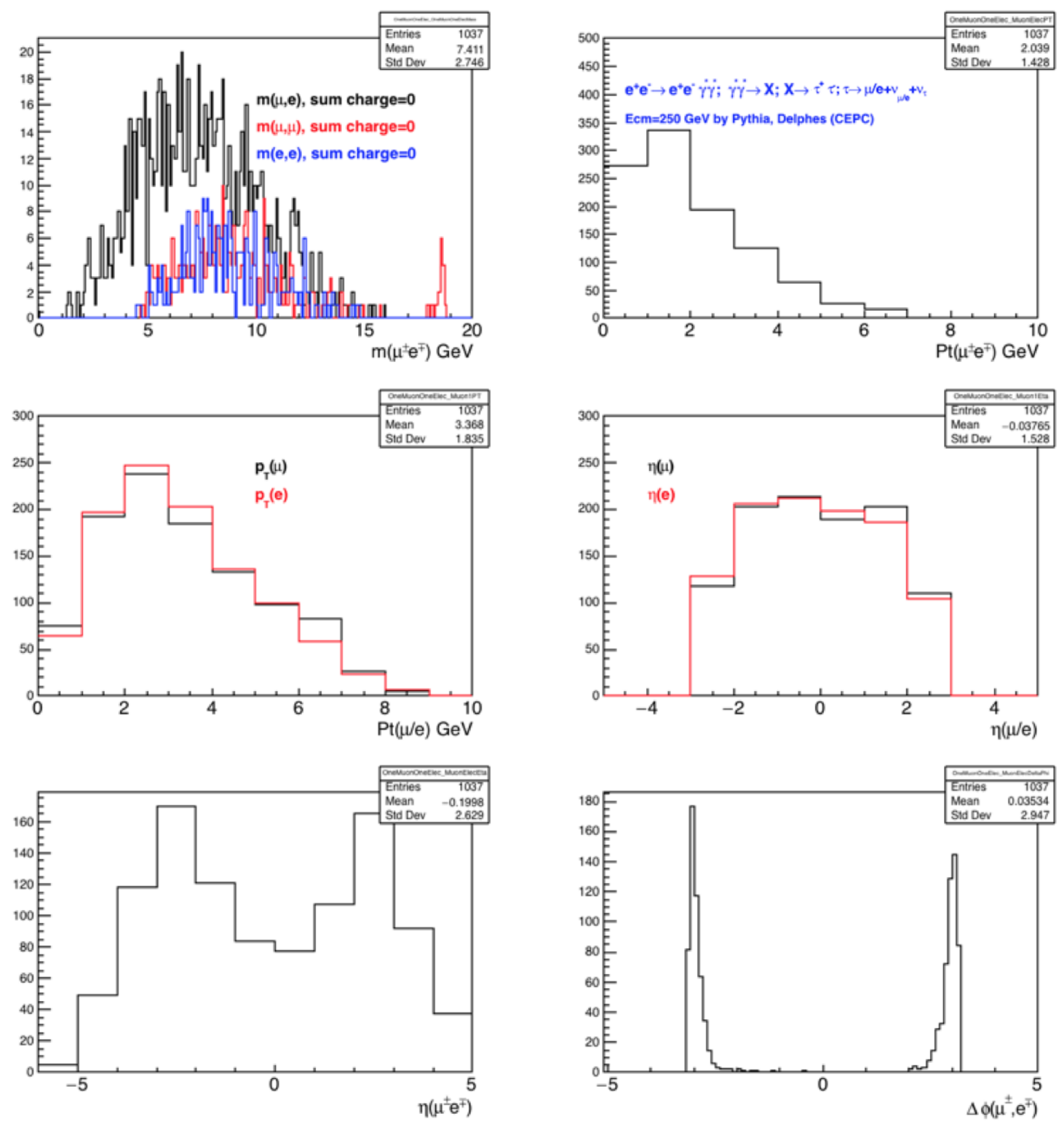

FIG. 10: The features of the $\mu^{ \pm} e^{\mp}\left(\tau^{ \pm} \tau^{\mp}\right)$ system in the $e^{+} e^{-} \rightarrow e^{+} e^{-} X ; X \rightarrow \tau^{ \pm} \tau^{\mp} ; \tau^{ \pm} \rightarrow \ell^{ \pm}+$anything - else, $\tau^{\mp} \rightarrow$ $\ell^{\mp}+$ anything - else, where $\ell=\mu, e$ process at CEPC: (top left) The $\mu^{ \pm} e^{\mp} / \mu^{ \pm} \mu^{\mp} / e^{ \pm} e^{\mp}$ mass distributions; (top right) The $p_{T}$ distributions of $\mu^{ \pm} e^{\mp}$; (middle left) The $p_{T}$ distribution of muon and electron; (middle right) The $\eta$ distribution of muon and electron; (bottom left) The $\eta$ distribution of $\mu^{ \pm} e^{\mp}$; (bottom right) The $\Delta \phi$ distribution between muon and electron. These events are simulated by Delphes with CEPC configuration. 


\section{OUTLOOK-A FIRST LOW ENERGY PHOTON COLLIDER SOMEWHERE}

A detail overview about two-photon physics can be found at Ref. [22, which summarizes the two-photon process at various colliders including possible photon colliders. There are many benefits to study states with spin 0 and spin 2 particles via a photon collider such as high luminosity, studying possible CP violation through polarized photons etc. The kinematic distributions for various decays are almost the same as that in the two-photon process at CEPC. As an example, we use this process: $\gamma \gamma \rightarrow X ; X \rightarrow Y(1 S) \mu^{+} \mu^{-} ; Y(1 S) \rightarrow \mu^{+} \mu^{-}$to demonstrate its kinematic distributions as shown in Fig. 11, where the total colliding energy is $18.5 \mathrm{GeV}$. The author is looking for possible collaboration to extend this section in the future.
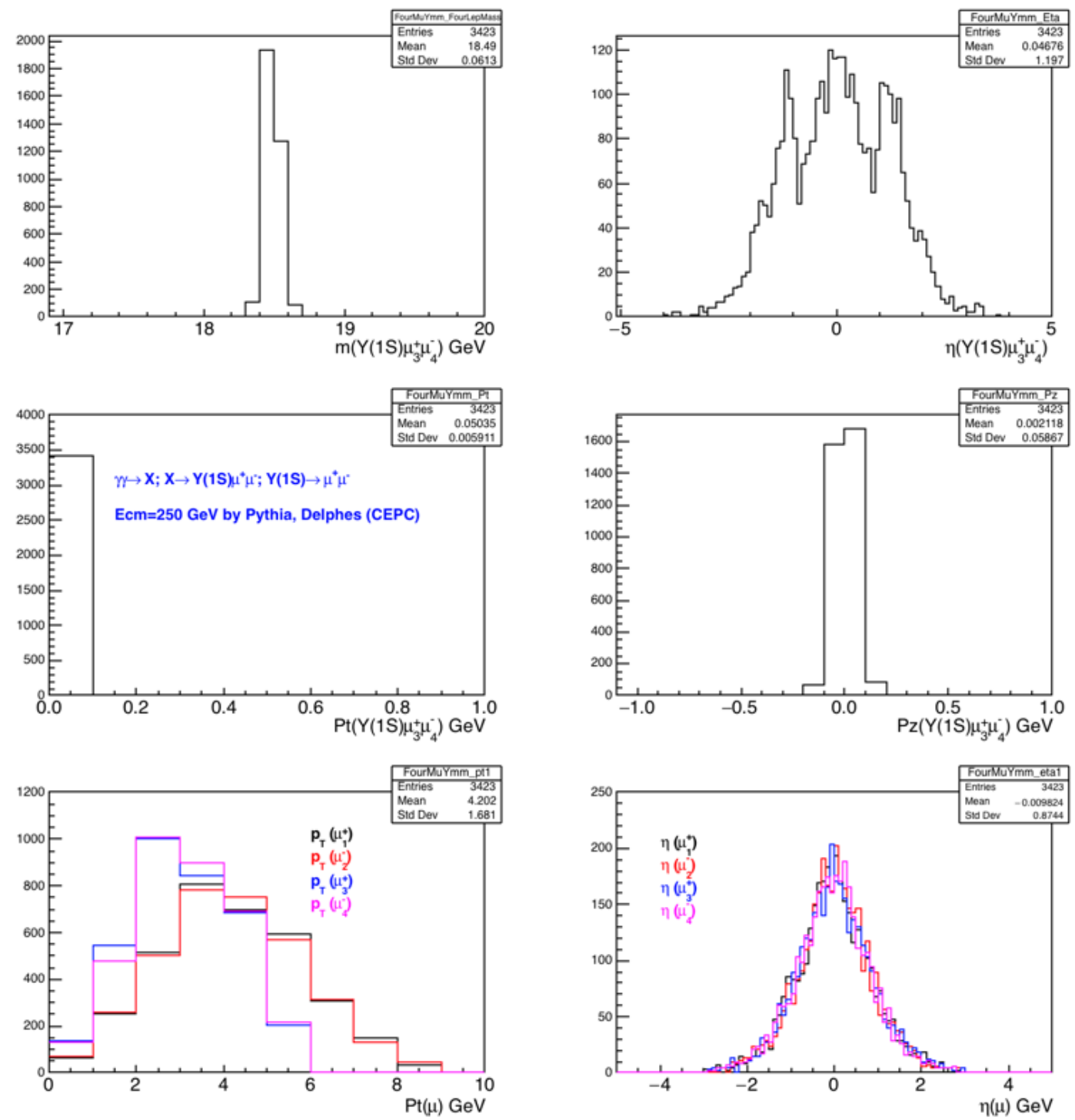

FIG. 11: The features of the four-muon system in a visionary photon collider with total energy of $18.5 \mathrm{GeV}(\gamma \gamma \rightarrow X ; X \rightarrow$ $Y(1 S) \mu^{+} \mu^{-} ; Y(1 S) \rightarrow \mu^{+} \mu^{-}$) (top left) The invariant mass; (top right) The $\eta$ distribution; (middle left) $p_{T}$; (middle right) $p_{Z}$; (bottom left) $p_{T}$ of the four muons. (bottom right) $\eta$ of the four muons. These events are simulated by Delphes with CEPC configuration. 


\section{Acknowledgments}

I would like to thank Stephen Mrenna, Stefan Prestel for their many detailed help on the usage of Pythia. All opinions and comments expressed, and any error committed, are solely responsibility of the author himself.

[1] CDF Collaboration, Phys. Rev. Lett. 102, 242002 (2009); CDF Collaboration, arXiv:1101.6058 [Hep-ex], Mod. Phys. Lett. A 321750139 (2017).

[2] Kai Yi, Int. J. Mod. Phys. A 28, 1330020 (2013).

[3] CMS Collaboration, Phys. Lett. B 734, 261 (2014).

[4] D0 Collaboration, Phys. Rev. D 89, 012004 (2014); D0 Collaboration, Phys. Rev. Lett. 115, 232001 (2015).

[5] LHCb Collaboration, Phys. Rev. D 95, 012002 (2017); LHCb Collaboration, Phys. Rev. Lett. 118, 022003 (2017).

[6] BaBar Collaboration, Phys. Rev. D 91, 032002 (2015); BES Collaboration, Phys. Rev. D 91, 012003 (2015).

[7] Belle Collaboration, Phys. Rev. Lett. 104112004 (2010).

[8] CMS Collaboration, Phys. Lett. B 764, 66 (2017).

[9] LHCb Collaboration, Phys. Rev. Lett. 107082002 (2011); CMS Collaboration, JHEP 1409, 094 (2.14); ATLAS Collaboration,Eur. $\quad$ Phys. J. $\quad$ C 7776 (2017); LHCb Collaboration, JHEP 1706, 047 (2.17); Erratum: JHEP 1710 068 (2017).

[10] CMS Collaboration, JHEP 1705, 013 (2017).

[11] Kamuran Dilsiz, University of Iowa, PhD thesis, http://ir.uiowa.edu/etd/3072/

[12] S. Durgut, Evidence of a Narrow Structure in $Y(1 S) l l$ Channel and CMS Phase I and II Silicon Detector Upgrade Studies, PhD thesis at University of Iowa; S. Durgut, Search for Exotic Mesons at CMS, APS April Meeting 2018, http://meetings.aps.org/Meeting/APR18/Session/U09.6

[13] C. Patrignani et al. (Particle Data Group), Chin. Phys. C, 40, 100001 (2016).

[14] M. Karliner et al., arXiv:1711.10626 [hep-ph].

[15] D. Cassidy and A. Mills Jr., Nature 449, 195 (2007).

[16] A. V. Berezhnoy, A. V. Luchinsky et al., Phys. Rev. D 86, 034004 (2012); M. Karliner, S. Nussinov et al., Phys. Rev. D95 (2017) 034011, arXiv:1611.00348; Y. Bai, S. Lu et al., arXiv:1612.00012 Z.-G. Wang, Eur. Phys. J. C77 (2017) 432, arXiv:1701.04285 J.-M. Richard, A. Valcarce et al., Phys. Rev. D95 (2017) 054019, arXiv:1703.00783 M. N. Anwar, J. Ferretti et al., arXiv:1710.02540 E. Eichten and Z. Liu, arXiv:1709.09605 R. Vega-Morales, arXiv:1710.02738, Y. Chen and R. Vega-Morales, arXiv:1710.02738 [hep-ph]; W. Chen, H. Chen et al., Phys.Lett. B773 (2017) 247-251; J. Wu, W. liu et al., Phys. Rev. D 97, 094015 (2018); W. Chen, H. Chen et al., arXiv:1803.02522 [hep-ph].

[17] C. Hughes, E. Eichten et al., Phys.Rev. D97 (2018) no.5, 054505.

[18] http://cepc.ihep.ac.cn/index.html

[19] https://twiki.cern.ch/twiki/bin/view/CMSPublic/LumiPublicResults

[20] T. Sjstrand, S. Mrenna et al., JHEP 05026 (2006); T. Sjstrand, S. Mrenna et al., Comput. Phys. Comm. 178 852 (2008); arXiv:0710.3820.

[21] The DELPHES 3 collaboration, JHEP 02, 057 (2014); arXiv:1307.6346 [hep-ex].

[22] I., Ginzburg, arXiv:1508.06581 [hep-ph]. 